\title{
Research on Mixed Measurement of Zero Sequence Parameters of Parallel Double Circuit Transmission Lines
}

\author{
Bin Shu, Pu Zhang \\ Beijing Electrical Economy and Technology Research Institute, Beijing 100055, China \\ Shengsuo Niu \\ Department of Electrical Engineering, North China Electric Power University, Baoding, China \\ Xiaoxiao Yu \& Li Ju \\ Beijing Electrical Economy and Technology Research Institute, Beijing 100055, China
}

\begin{abstract}
For the measurement of zero sequence parameters of the parallel double circuit transmission line, the conventional off-line measurement method cannot meet the need of the zero sequence parameter measurement, this paper presents a hybrid measurement method, by applying a zero-sequence signal on line outage, simultaneous measuring three double-circuit transmission line voltage and current. Accurate measurement of zero sequence parameters and mutual parameters of double circuit transmission lines can be achieved. The simulation results verify the effectiveness of the method.
\end{abstract}

KEYWORD: transmission line; zero sequence parameters; mixing measuring

\section{INTRODUCTION}

The power flow calculation, short-circuit current calculation, relay protection and fault analysis will use the transmission line parameters. Accurate measurement of the parameters of transmission lines is of great significance for the analysis and calculation of the power system.

The measurements of line parameter is usually in front of the new line put into operation. At present, most of the engineering practice uses off-line single ended measurement method. For the same tower double circuit line, in the case of two circuit line outage, power signals are applied in two circuit lines, measuring zero sequence parameters and zero sequence mutual parameters. In some cases, such as: in the same tower has built a double-circuit line, parameter measurement for the new line needs to be run under the old line state; or line switching occurs transformation, then it also need to re-measure parameters. In order to ensure reliability of power supply, usually to ensure that one of the double circuit line in a running back, another in the back line outage condition measurement parameters. For the parameter measurement and engineering generally do not measure the mutual inductance parameters between the two lines, measured in a back line zero sequence inductance parameter does not considering another loop, resulting in zero sequence parameter measurement inaccurate and incomplete. This paper proposes a method for measuring the mixed transmission line parameters, which can effectively solve this problem.

\section{PRINCIPLE OF MIXED MEASUREMENT METHOD FOR ZERO SEQUENCE PARAMETERS OF PARALLED DOUBLE CIRCUIT TRANSMISSION LINE}

As shown in FIG.1, mixed measurement that is, considering one back to the normal operation, another parameter measurements outage time. I line running in their systems, at both ends of the PT, CT secondary side access two PMU, used to measure the threephase voltage and phase current. II line outage, power is applied to test the $\mathrm{N}$ terminal. II line $\mathrm{M}$ terminal connection mode according to different tests are different. II line in the $\mathrm{N}$ terminal access a PMU, based on the measurement needs adding PT, CT or utilization system PT, CT.

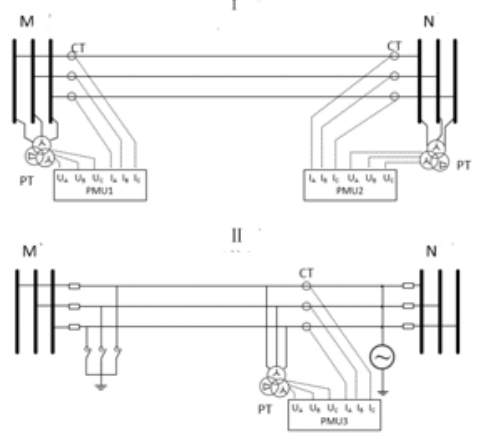

Figure1. Parallel double-circuit line zero-sequence parameter measurement wiring diagram 
Corresponding zero-sequence parameters of the model shown in Figure 2.

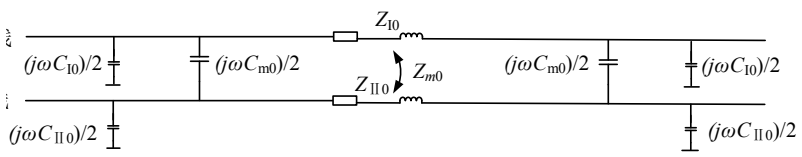

Figure2. Parallel double-circuit line zero-sequence parameters of the model

\subsection{Zero Sequence Impedance Parameters Measurement}

As shown in Figure 1, the ground isolation switch of II line $M$ terminal is closed, Single-phase power is applied at $\mathrm{M}$ terminal. The $220 \mathrm{~V}$ power supply can be provided by the low voltage side of the transformer station. PMU1, PMU2 and PMU3 simultaneous measure the entire three-phase voltage and current, calculating throughout the zero-sequence voltage and zero-sequence current signal: $\dot{U}_{10}, \dot{U}_{20}$, $\dot{U}_{30}, \dot{I}_{10}, \dot{I}_{20}, \dot{I}_{30}$.Subscript 1, 2, 3 indicates the PMU number and the subscript 0 indicates the zero sequence. Because the zero sequence voltage is very low, the current of zero-sequence capacitance to ground and mutual zero sequence capacitor is very small .It can be ignored. According to equation (1) can be obtained as in Fig.2.

$$
\left\{\begin{array}{c}
\dot{U}_{30}=\dot{I}_{30} Z_{\mathrm{II} 0}+\left(\frac{\dot{I}_{20}-\dot{I}_{10}}{2}\right) Z_{m 0} \\
\dot{U}_{20}-\dot{U}_{10}=\dot{I}_{30} Z_{m 0}+\left(\frac{\dot{I}_{20}-\dot{I}_{10}}{2}\right) Z_{\mathrm{I0}}
\end{array}\right.
$$

In the equation, $Z_{\mathrm{I} 0}, Z_{\mathrm{II} 0}$ represents zero sequence self-impedance ofIand II line. $Z_{m 0}$ represents zero sequence mutual impedance between theIand II line.

If the erected parallel double-circuit line have the same, that is $Z_{\mathrm{I} 0}=Z_{\mathrm{II} 0}$, it can solve the zero sequence impedance parameters and mutual impedance parameters between I and II lines by formula (1).

If the parameters of erected parallel doublecircuit line are not the same, that is $Z_{\mathrm{I} 0} \neq Z_{\mathrm{II} 0}$, you can do line parameter measurement test by rotation stoppingI and II line., Apply zero-sequence signal to line I, swapp measuring equipment for two lines, and then do a similar test measuring a set of data in line I outage situation, that is $\dot{U}_{10}^{\prime}, \dot{U}_{20}^{\prime}, \dot{U}_{30}^{\prime}, \dot{I}_{10}^{\prime}$, $\dot{I}_{20}^{\prime}, \dot{I}_{30}^{\prime}$. It can obtain the line I and II impedance parameters and zero-sequence mutual impedance parameters by formula (2) equations.

$$
\left\{\begin{array}{c}
\dot{U}_{30}=\dot{I}_{30} Z_{110}+\left(\frac{\dot{2}_{20}-\dot{I}_{10}}{2}\right) Z_{m 0} \\
\dot{U}_{20}-\dot{U}_{10}=\dot{I}_{30} Z_{m 0}+\left(\frac{\dot{I}_{20}-\dot{I}_{10}}{2}\right) Z_{10} \\
\dot{U}_{30}^{\prime}=\dot{I}_{30}^{\prime} Z_{10}+\left(\frac{\dot{I}_{20}^{\prime}-\dot{I}_{10}^{\prime}}{2}\right) Z_{m 0} \\
\dot{U}_{20}^{\prime}-\dot{U}_{10}^{\prime}=\dot{I}_{30}^{\prime} Z_{m 0}+\left(\frac{\dot{I}_{20}^{\prime}-\dot{I}_{0}^{\prime}}{2}\right) Z_{110}
\end{array}\right.
$$

\subsection{Zero sequence admittance parameter measurement}

Reference to Fig.1, it can open circuit the Mterminal of line I, remove applied power on threephase line $\mathrm{N}$ terminal, shunt $\mathrm{N}$ terminal of threephase, switch a phase of $\mathrm{N}$ terminal together on the line, apply three-phase line with a phase system voltage. Simultaneous measure the three-phase voltage and current by PMU, calculating zero sequence parameter: $\dot{U}_{10}, \dot{U}_{20}, \dot{U}_{30}, \dot{I}_{10}, \dot{I}_{20}, \dot{I}_{30}$. Due to II test line open at such state, the current flow is very small, and the first terminal voltage is approximately equal. According to FIG. 2 can construct equations (3) as shown in the formula.

$$
\left\{\begin{array}{c}
\left(j \omega C_{\mathrm{II}}\right) \dot{U}_{3}+\left(j \omega C_{m 0}\right)\left(\dot{U}_{3}-\frac{\dot{U}_{1}+\dot{U}_{2}}{2}\right)=\dot{I}_{3} \\
\left(j \omega C_{\mathrm{I} 10}\right)\left(\frac{\dot{U}_{1}+\dot{U}_{2}}{2}\right)+\left(j \omega C_{m 0}\right)\left(\frac{\dot{U}_{1}+\dot{U}_{2}}{2}-\dot{U}_{3}\right)=\frac{\dot{I}_{1}+\dot{I}_{2}}{2}
\end{array}\right.
$$

If the double-circuit line have the same parameter, that is $C_{\mathrm{I} 0}=C_{\mathrm{II} 0}$, it can solute the zero-sequence capacitance parameters and zero-sequence mutual capacitance between Iline and II line by the formula (3).

If the double-circuit line self-parameters is not the same, that is $C_{\mathrm{I} 0} \neq C_{\mathrm{II} 0}$, you can do line parameter measurement test by rotation stoppingI and II line., Apply zero-sequence signal to line I, swapp measuring equipment for two lines, and then do a similar test measuring a set of data in line I outage situation, that is $\dot{U}_{10}^{\prime}, \dot{U}_{20}^{\prime}, \dot{U}_{30}^{\prime}, \dot{I}_{10}^{\prime}, \dot{I}_{20}^{\prime}, \dot{I}_{30}^{\prime}$. Through building formula (4) equations, solve the available line I and II self-capacitance parameters and zero-sequence mutual capacitance parameters.

$$
\left\{\begin{array}{c}
\left(j \omega C_{\mathrm{II}}\right) \dot{U}_{3}+\left(j \omega C_{m 0}\right)\left(\dot{U}_{3}-\frac{\dot{U}_{1}+\dot{U}_{2}}{2}\right)=\dot{I}_{3} \\
\left(j \omega C_{\mathrm{I0}}\right)\left(\frac{\dot{U}_{1}+\dot{U}_{2}}{2}\right)+\left(j \omega C_{m 0}\right)\left(\frac{\dot{U}_{1}+\dot{U}_{2}}{2}-\dot{U}_{3}\right)=\frac{\dot{I}_{1}+\dot{I}_{2}}{2} \\
\left(j \omega C_{10}\right) \dot{U}_{3}^{\prime}+\left(j \omega C_{m 0}\right)\left(\dot{U}_{3}^{\prime}-\frac{\dot{U}_{1}^{\prime}+\dot{U}_{2}^{\prime}}{2}\right)=\dot{I}_{3}^{\prime} \\
\left(j \omega C_{\mathrm{II}}\right)\left(\frac{\dot{U}_{1}^{\prime}+\dot{U}_{2}^{\prime}}{2}\right)+\left(j \omega C_{m 0}\right)\left(\frac{\dot{U}_{1}^{\prime}+\dot{U}_{2}^{\prime}}{2}-\dot{U}_{3}^{\prime}\right)=\frac{\dot{I}_{1}^{\prime}+\dot{I}_{2}^{\prime}}{2}
\end{array}\right.
$$

After solving the above parameters, divided by the length of the ine to get the zero sequence line parameters per unit length.

\section{SIMULATION}

To verify the effectiveness of the proposed method, it can make a simulation in MATLAB / Simulink environment. The simulation model structures are shown in Figure 3. 


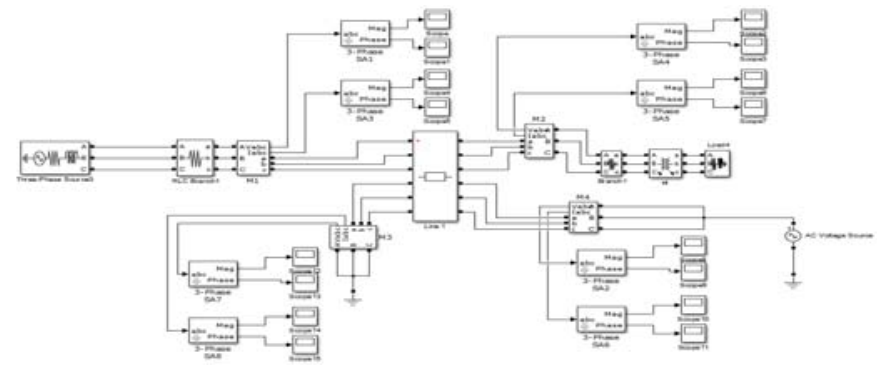

Figure 3. Parallel double-circuit line zero-sequence parameter measurement MATLAB Simulation Model

Line voltage level is $220 \mathrm{kV}$, double circuit line of the same model. The length of line is $25 \mathrm{~km}$. Line parameter settings are shown in Table 1.

TABLE 1. Simulation of double-circuit line zero-sequence parameters settings

\begin{tabular}{c|c|c|c|c|c}
\hline $\begin{array}{c}R_{\mathrm{I} 0}=R_{\Pi 1} \\
(\Omega / \mathrm{km})\end{array}$ & $\begin{array}{c}R_{\mathrm{m} 0} \\
(\Omega / \mathrm{km})\end{array}$ & $\begin{array}{c}L_{\mathrm{I} 0}=L_{\Pi 1} \\
(\mathrm{mH} / \mathrm{km})\end{array}$ & $\begin{array}{c}L_{\mathrm{m} 0} \\
(\mathrm{mH} / \mathrm{km})\end{array}$ & $\begin{array}{c}C_{\mathrm{I} 0}=C_{\mathrm{II} 0} \\
(\mathrm{~F} / \mathrm{km})\end{array}$ & $\begin{array}{c}C_{\mathrm{m} 0} \\
(\mathrm{~F} / \mathrm{km})\end{array}$ \\
\hline 0.174 & 0.134 & 2.613 & 1.134 & $6.34 \mathrm{e}-9$ & $1.50 \mathrm{e}-9$ \\
\hline
\end{tabular}

The result of parameter measurement and the relative error are shown in table 2.

TABLE2. Measurement value and error of zero sequence parameters of double circuit lines

\begin{tabular}{l|l|l|l|l|l|l}
\hline & $\begin{array}{l}R_{\mathrm{I} 0}=R_{\Pi 1} \\
(\Omega / \mathrm{km})\end{array}$ & $\begin{array}{l}R_{\mathrm{m} 0} \\
(\Omega / \mathrm{km})\end{array}$ & $\begin{array}{l}L_{\mathrm{I} 0}=L_{\Pi 0} \\
(\mathrm{mH} / \mathrm{km})\end{array}$ & $\begin{array}{l}L_{\mathrm{m} 0} \\
(\mathrm{mH} / \mathrm{km})\end{array}$ & $\begin{array}{l}C_{\mathrm{I} 0}=C_{\Pi 1} \\
(\mathrm{~F} / \mathrm{km})\end{array}$ & $\begin{array}{l}C_{\mathrm{m} 0} \\
(\mathrm{~F} / \mathrm{km})\end{array}$ \\
\hline & 0.1745 & 0.1346 & 2.6156 & 1.1352 & $6.341 \mathrm{e}-9$ & $1.496 \mathrm{e}-9$ \\
\hline & 0.29 & 0.45 & 0.10 & 0.11 & 0.02 & -0.27 \\
\hline
\end{tabular}

Simulation of the resulting zero-sequence line parameter measurement error is less than $0.5 \%$, whereby the validity of the proposed method.

\section{CONCLUSION}

For double circuit transmission lines on the same tower and frame, such as in the event of a line cut change, etc. need to be re-measured parameters, but because of the power supply reliability requirements, generally is not allowed to double-circuit line outage. The transmission line parameters of mixed measurement methods described in this paper, in which a back line running condition test double circuit zero sequence parameters and mutual parameters. Ensuring the reliability of power supply while achieving double-circuit line accurate measurement of zero-sequence parameters, simulation results demonstrate the effectiveness of the method. The proposed measurement method has practical engineering value.

\section{REFERENCES}

Chen X. 2007. Line measurement of positive, negative and zero sequence parameters of transmission lines with mutual inductance, Power Engineering Conference:1337-1342.

Hu Zhijian \& Liu Meiguan. 1999. Research and implementation of parameter line parameter measurement. Automation of Electric Power Systems, 23(24):32-35.

Liang Zhirui. 2013. Coupled double-circuit transmission line zero-sequence parameters on-line measurement. Electric Power Automation Equipment, 33(7):70-74.

Hu Zhijian \& Liu Meiguan. 1999. Research and implementation of parameter line parameter measurement. Automation of Electric Power Systems, 23(24):32-35.

Zhijian H. 2004. New live line measurement methods of transmission lines inductance parameters based on GPS, Power System Technology:63-68. 